\title{
Contralateral C-7 transfer: is direct repair really superior to grafting?
}

\author{
Anil Bhatia, MD, ${ }^{1}$ Piyush Doshi, MD, ${ }^{2}$ Ashok Koul, MD, ${ }^{3}$ Vitrag Shah, MD, ${ }^{4}$ Justin M. Brown, MD, ${ }^{5}$ \\ and Mahmoud Salama, MD 6
}

\begin{abstract}
${ }^{1}$ Department of Brachial Plexus Surgery, Deenanath Mangeshkar Hospital, Pune; ${ }^{2}$ Department of Plastic Surgery, SBKS Medical Institute and Research Centre, Vadodara; ${ }^{3}$ Department of Plastic Surgery, Sparsh Hospital, Bengaluru; ${ }^{4}$ Department of Plastic Surgery, Shah Hospital, Surat, India; ${ }^{5}$ Department of Neurosurgery, UC San Diego Health, San Diego, California; and ${ }^{6}$ Department of Orthopaedics, Aswan University, Aswan, Egypt
\end{abstract}

It is not uncommon for a severe traumatic brachial plexus injury to involve all 5 roots, resulting in a flail upper limb. In such cases, surgical reconstruction is often palliative, providing only rudimentary function. Nerve transfers are the mainstay of reconstructive strategies due to the predominance of root avulsions. Consistent results are obtained only for restoration of shoulder stability and elbow flexion, whereas restoring useful hand function remains a challenge. The transfer of the contralateral C-7 (cC-7) is commonly used in an attempt to restore basic hand function, but results are notoriously unreliable and inconsistent. Shu-feng Wang and colleagues recently proposed a potentially more successful permutation of this procedure. They advocated direct approximation of the $\mathrm{CC}-7$ to the lower trunk on the paralyzed side, thus avoiding the interposition of nerve grafts. This technique involves a lengthy dissection of the $\mathrm{CC}-7$ transfer across the midline via a prespinal route, as well as extensive mobilization of the ipsilateral lower trunk by cutting a subset of its branches, adducting the arm, and (if necessary) shortening the humerus. Each of these steps is indispensable to achieve direct approximation of the nerve ends. Many surgeons have tried to emulate Wang's strategy. However, the technical difficulties involved have forced recourse to interposition of nerve grafts once again.

The authors report their observations in the first 22 patients in whom they performed this procedure. Direct cC-7 repair via the prespinal route was performed in 12 patients. Shortening of the humerus was necessary in 9 of these 12 patients. In 10 patients, a direct repair was not feasible and nerve grafting was performed. The median follow-up period was 26 months for the direct coaptation group and 28.5 months for the nerve graft group.

In the direct repair group, 10 of the 12 patients regained Medical Research Council Grade 3 flexion of the wrist and of the middle, ring, and little fingers, while the remaining 2 patients had Grade 2 function. Flexion appeared 12-14 months after the operation. At the latest follow-up, these patients could activate the wrist and hand without requiring significant augmentation maneuvers in the donor limb. In contrast, repair requiring interposition grafts resulted in Grade 3 strength in only 2 of 10 patients, while 7 had Grade 2 strength, and 1 experienced failure. In all grafted cases, the patient had to forcibly contract the contralateral pectoralis major and triceps muscles to produce the weak movements on the reconstructed side.

In this small series, the authors demonstrated a distinct advantage associated with the avoidance of grafts when transferring the $\mathrm{cC}-7$ to restore hand function. The authors conclude that efforts to achieve direct approximation of the donor C-7 and the recipient lower trunk are necessary and justified.

https://thejns.org/doi/abs/10.3171/2017.4.FOCUS1794

KEY WORDS nerve transfer; contralateral C-7; interposition graft; direct coaptation; prespinal route

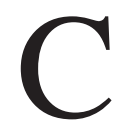
LOSED traction injuries of the brachial plexus are becoming increasingly frequent, and cases affecting all 5 roots are not uncommon. Such patients present with complete loss of function from the shoulder to the fingers since the upper limb has been neurologically disconnected from the spinal cord. The arm and hand hang lifeless from the shoulder, and any meaningful restoration of function depends on surgical reconstruction.

The treatment of complete brachial plexus palsies has many limitations. ${ }^{14,19}$ The overwhelming paucity of axon

ABBREVIATIONS cC-7 = contralateral C-7; MRC = Medical Research Council.

SUBMITTED March 1, 2017. ACCEPTED April 19, 2017.

INCLUDE WHEN CITING DOI: 10.3171/2017.4.FOCUS1794. 


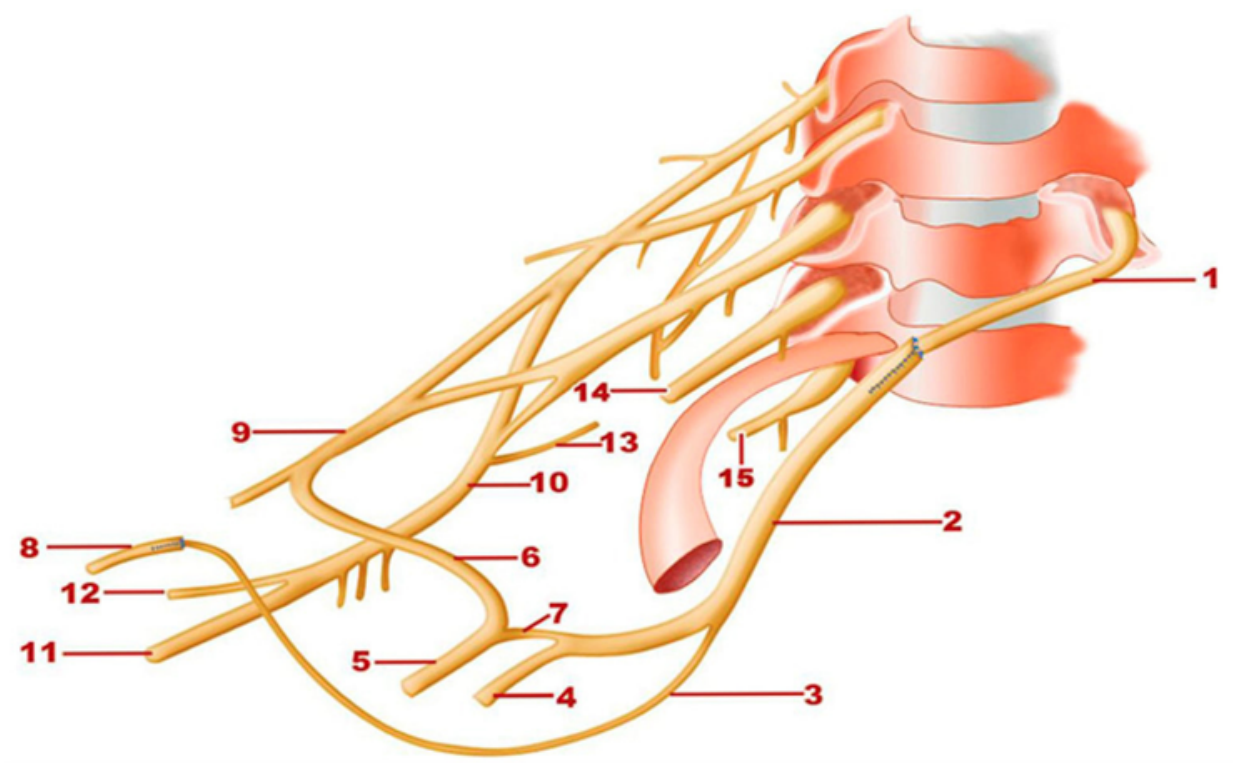

FIG. 1. Schematic the basic concepts of the transfer. The $\mathrm{cC}-7$ (1) is cut as long as possible and transferred via the prespinal route to the contralateral scalene. The ipsilateral lower trunk (2) is mobilized proximally for direct repair to this structure. The posterior division of the lower trunk is divided to allow proximal mobilization and to direct axons primarily to the anterior division and thus wrist and finger flexion. The medial cutaneous nerves (3) are also cut to assist in this mobilization and then transferred to the musculocutaneous nerve (8) to provide innervation to elbow flexors as well. $4=$ median nerve; $5=$ ulnar nerve; $6=$ lateral root of the median nerve; $7=$ medial root of the median nerve; $9=$ lateral cord; $10=$ posterior cord; $11=$ radial nerve; $12=$ axillary nerve; $13=$ posterior division of the lower trunk; $14=\mathrm{C}-8$ nerve root; $15=\mathrm{T}-1$ nerve root. Reprinted with permission from Wang SF, Li PC, Xue YH, Yiu HW, Li YC, Wang HH: Contralateral C-7 nerve transfer with direct coaptation to restore lower trunk function after traumatic brachial plexus avulsion. J Bone Joint Surg Am 95:821-827, 2013, http://journals.Iww.com/jbjsjournal/pages/default.aspx.

donors and the uncertain results following extensive reconstructions have proved to be major deterrents of surgical endeavors. Experience with extraplexus nerve transfers has allowed us to regain certain functions at the shoulder and elbow in a more consistent manner. ${ }^{15,21}$ However, restoration of effective prehension remains elusive. Several creative strategies have been proposed to achieve this end. These have included intercostal transfers to the median nerve or to a transplanted gracilis muscle. ${ }^{1}$ Another option is thoracoscopic harvest of the phrenic nerve for direct approximation to the median nerve..$^{25} \mathrm{~A}$ distal-to-proximal reconstruction strategy, as proposed by Doi et al., aims to use the proximal donor nerves to innervate free functioning muscle transfers. These transfers are primarily used to achieve flexion and extension of the fingers, along with flexion and extension of the elbow. ${ }^{4,5}$ However, the results of Doi et al. have not been replicated by other groups.

$\mathrm{Gu}$ et al. introduced an important new concept when they described the use of the contralateral C-7 (cC-7) for restoration of function in the median nerve territory in $1986 .{ }^{8}$ However, this method has not been adopted widely for several reasons, including the fear of creating a deficit in the intact opposite upper limb, the long delay in the appearance of function, and the weak strength of restored finger flexion. It has also been commonly noted that the patient has to perform forcible maneuvers with the opposite arm to demonstrate the recovered muscle actions. Therefore, independent use of the affected hand is not possible. A systematic review of the literature has showcased the difficulty in judging the utility of the cC-7 transfer. ${ }^{26}$ The use of part of versus the entire root, different methods of harvesting the C-7 root, use of vascularized or nonvascularized nerve grafts, subcutaneous or prespinal passage, and whether a single function or multiple functions are targeted all contribute to the wide variety of reported outcomes. $3,8,16,17$

According to Wang et al., ${ }^{24}$ the secret to achieving better hand function is direct approximation of the full cC-7 to the anterior division of the lower trunk on the paralyzed side. The posterior division of the lower trunk is separated to avoid concomitant innervation of antagonist muscles. This approach has raised several questions. Successful direct coaptation depends on challenging technical innovations with their inherent risks to mobilize both the cC-7 and the target lower trunk to a position where they directly reach one another. On the injured side, the pectoral and cutaneous branches of the lower trunk must be sectioned and the posterior division of the lower trunk has to be separated, leaving the medial cord as the functional recipient. Similarly, the harvest of the $\mathrm{cC}-7$ has to be carried as distally as possible, including separation of the posterior divisions as they converge to form the posterior cord. The cC-7 is divided as distally as possible and then passed across the midline through the retropharyngeal space just anterior to the cervical spine. This can typically bring it to a position just medial to the anterior scalene muscle on the injured side (Fig. 1). To provide additional length (but adding to the technical challenges and risks of this procedure), Wang et al. advocate passage deep to the contralateral vertebral artery. ${ }^{24}$ Finally, tension on the repair between $\mathrm{cC}-7$ and ipsilateral anterior division of the lower trunk is eased by dramatic adduction of the recipi- 

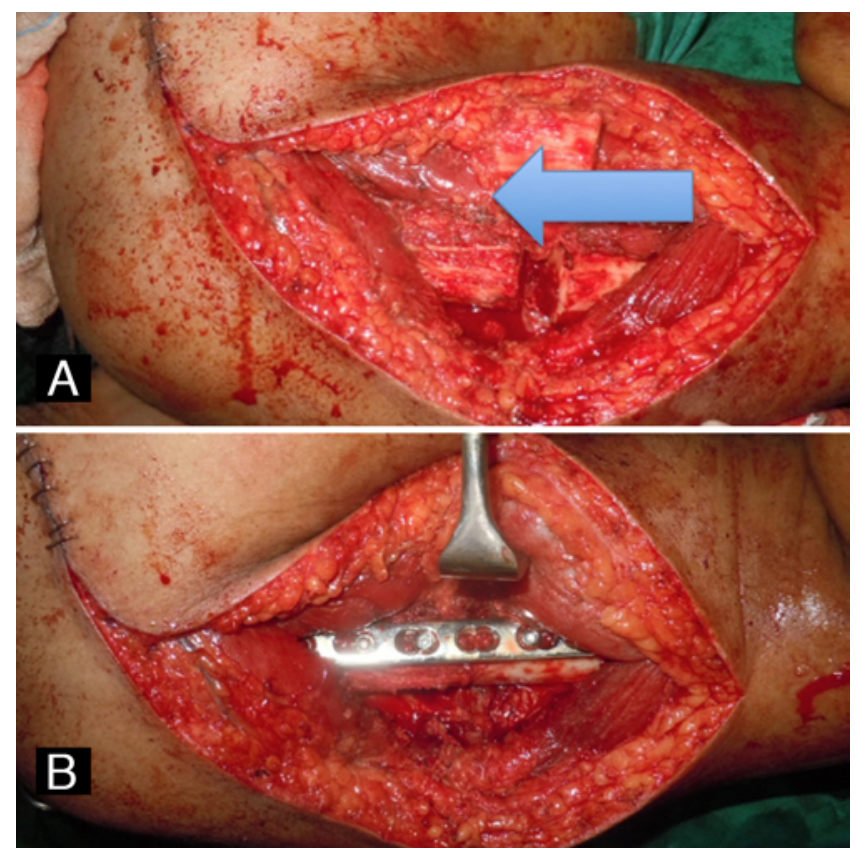

FIG. 2. A: Intraoperative photograph showing resection of 2.5 to $3.5 \mathrm{~cm}$ of the proximal humerus and approximation of the bone ends (arrow). B: After humeral shortening, a dynamic compression plate is used to fixate the humerus at the new shortened length to shorten the distance from the lower trunk to midline.

ent arm, which, if unsuccessful in allowing the nerves to reach one another, is augmented by a judicious shortening osteotomy of the humerus (Fig. 2).

Since October 2013, we have adopted the aforementioned strategy described by Wang et al. for the treatment of traumatic complete brachial plexus injuries with avulsions of all 5 roots. Inclusion criteria were patient age younger than 35 years and presentation within 11 months (preferably 6 months) of the injury. We additionally excluded patients with other associated neurological injuries (e.g., spinal cord or brain), deforming injuries of the extremity, pulmonary compromise, contractures limiting joint range of motion in the limb, and vascular compromise of the extremity.

Because of the complexity of this technique, direct repair was more commonly abandoned in the earlier cases in favor of grafts. As we became more proficient, we had greater success in achieving a direct repair. Thus, we had a series of 12 patients in whom a direct repair between cC-7 and the ipsilateral lower trunk (the direct coaptation group) was done, and 10 patients in whom nerve grafting between the cC-7 and ipsilateral lower trunk was performed.

The object of the present study was to retrospectively evaluate the quality of reinnervation of the forearm flexors in the 2 groups and to determine whether the increased effort and risk required for achieving a direct repair was warranted.

\section{Methods}

Since October 2013, cC-7 transfer using the entire root has been used in the treatment of 43 patients. All of these
TABLE 1. Characteristics of patients in the direct coaptation group

\begin{tabular}{ccccc}
\hline $\begin{array}{c}\text { cC-7 Direct } \\
\begin{array}{c}\text { Coaptation } \\
\text { Group }\end{array}\end{array}$ & $\begin{array}{c}\text { Age } \\
\text { (yrs) }\end{array}$ & $\begin{array}{c}\text { Preop } \\
\text { Denervation } \\
\text { Time (mos) }\end{array}$ & $\begin{array}{c}\text { Follow-Up } \\
\text { (mos) }\end{array}$ & $\begin{array}{c}\text { Finger \& Wrist } \\
\text { Flexion MRC } \\
\text { Grade }\end{array}$ \\
\hline $1^{*}$ & 28 & 2 & 36 & 3 \\
\hline 2 & 24 & 2 & 39 & 3 \\
\hline $3^{*}$ & 23 & 3 & 24 & 3 \\
\hline 4 & 30 & 1 & 31 & 2 \\
\hline $5^{*}$ & 23 & 3 & 33 & 3 \\
\hline 6 & 17 & 1 & 27 & 3 \\
\hline 7 & 31 & 3 & 30 & 3 \\
\hline 8 & 18 & 2.5 & 25 & 3 \\
\hline 9 & 20 & 5.5 & 23 & 2 \\
\hline 10 & 23 & 6 & 24 & 3 \\
\hline 11 & 35 & 3 & 24 & 3 \\
\hline 12 & 20 & 4 & 23 & 3 \\
\hline
\end{tabular}

* Shortening of the humerus was not required.

patients had suffered injuries to all 5 roots of the brachial plexus. The first 22 patients were selected for this retrospective study, as sufficient postoperative follow-up had been achieved. These included 12 patients in whom a direct repair was performed and 10 patients who underwent nerve grafting. Only 3 patients were female ( 2 in the direct group and 1 in the nerve grafting group). The median age for the direct coaptation group was 23 years (range 17-35 years), while for the nerve graft group it was 24 years (range 15-27 years). The median delay prior to surgery was 3 months (range 1-6 months) for the direct coaptation group and 3.5 months (range 1.5-11 months) for the nerve graft group. The demographic characteristics of the patients in the 2 groups are represented in Tables 1 and 2.

The patients were offered surgery on clinical grounds. In our practice, a high-velocity accident resulting in a complete deficit with positive Horner's sign and no indication of recovery in any muscle groups at more than 1 month from the accident is sufficient to warrant exploration and reconstruction based on the operative findings.

TABLE 2. Characteristics of patients in the nerve graft group

\begin{tabular}{ccccc}
\hline $\begin{array}{c}\text { cC-7 Nerve } \\
\text { Graft Group }\end{array}$ & $\begin{array}{c}\text { Age } \\
\text { (yrs) }\end{array}$ & $\begin{array}{c}\text { Preop } \\
\text { Denervation } \\
\text { Time (mos) }\end{array}$ & $\begin{array}{c}\text { Follow-Up } \\
\text { (mos) }\end{array}$ & $\begin{array}{c}\text { Finger \& } \\
\text { Wrist Flexion } \\
\text { MRC Grade }\end{array}$ \\
\hline 1 & 15 & 6.5 & 24 & 0 \\
\hline 2 & 24 & 1.5 & 35 & 3 \\
\hline 3 & 24 & 2 & 34 & 2 \\
\hline 4 & 21 & 2 & 36 & 2 \\
\hline 5 & 19 & 1.5 & 31.5 & 3 \\
\hline 6 & 24 & 2.5 & 29 & 2 \\
\hline 7 & 19 & 11 & 28 & 2 \\
\hline 8 & 27 & 4 & 26 & 2 \\
\hline 9 & 24 & 7 & 23 & 2 \\
\hline 10 & 27 & 6 & 26 & 2 \\
\hline
\end{tabular}


Whenever possible, MRI of the cervical spine was performed to help anticipate the levels of avulsion that would be encountered.

Electrodiagnostic studies were not uniformly performed. The information obtained from these studies has not proven helpful to our decision-making. At the time of exploration, root avulsions can be directly visualized, eliminating the ambiguity often provided by preoperative electrodiagnostic studies. Similarly, studies of the contralateral limb were determined to be unnecessary, as a limb with full strength and sensation that had not suffered any injuries at the time of the trauma would be expected to have completely normal findings. Patients whom we felt were candidates for the procedure were counseled preoperatively that, should they not have sufficient roots for grafting, we would proceed with the contralateral transfer as described. Final decision-making as to whether to proceed with this procedure was made at the time of the intervention based on the intraoperative findings.

\section{Operative Technique}

The operation is performed in the supine position with the neck extended (a pillow is placed under the patient at the level of the scapulae). The injured brachial plexus is exposed first. The exploration starts with isolation and stimulation of the phrenic nerve. The interscalene area is examined for ruptured root stumps. Their quality is examined by inspection of the cut section under the microscope and by stimulating the branches to the long thoracic nerve (from the C-5, C-6, and C-7 roots). When these contributions to the long thoracic nerve are functional, avulsion can be excluded. The ipsilateral lower trunk is traced proximally up to the C7-T1 and T1-2 intervertebral foramina, and the presence or absence of root avulsions is confirmed. When avulsion is confirmed, the avulsed roots are freed from local soft-tissue attachments, and the lower trunk is then delivered below the clavicle and dissection proceeds as follows, according to the technique of Wang et al. ${ }^{24}$ The pectoral branches associated with the medial cord are divided and the posterior division of the lower trunk is separated proximally. The posterior division can be neurolyzed away from the rest of the lower trunk, leaving only the anterior division without shortening either contribution. The medial cutaneous nerves to the arm and forearm are freed in the arm and divided distally. Next, the proximal stump of the remaining lower trunk is carefully inspected and trimmed until a healthy nerve is available. We send frozen sections and delay the final repair until the condition of the nerve end is confirmed. Finally, after this potential shortening of the nerve length, the arm is positioned across the chest, and the ability of the remaining lower trunk to reach the medial border of the ipsilateral anterior scalene muscle is assessed. If the reach appears adequate, but is within a couple centimeters, the utility of shortening of the humerus by $2.5-3.5 \mathrm{~cm}$ to achieve that reach is included in this calculation.

The opposite supraclavicular brachial plexus is then exposed via a similar approach. The phrenic nerve and the upper trunk are identified. The cC-7 is isolated and traced as distally as possible. At the level of the clavicle, where the $\mathrm{cC}-7$ or middle trunk splits into its anterior and pos- terior divisions, a pectoral branch is found exiting from the anterior division. The anterior and posterior divisions, including the pectoral branch, are then divided with care to protect the converging anterior division of the upper trunk as well as the converging posterior divisions of the upper and lower trunks. Wang et al. ${ }^{24}$ have highlighted the importance of the posterior division of the lower trunk and how damage to this structure will result in a finger extension deficit. Thus, particular care is taken while separating the posterior division of the middle trunk from that of the lower trunk. The mobilization of the root is completed by sectioning the proximal contribution to the long thoracic nerve, which, if left in place, would limit the nerve's reach to the contralateral side. Of note, the remaining C-5 and C-6 contributions to the long thoracic nerve are sufficient to avoid a serratus anterior muscle deficit. The $\mathrm{cC}-7$ is then delivered via the retropharyngeal space to the injured side and should come to rest just medial to the anterior scalene muscle on that side. The point of pivot on the side from which the nerve is transferred is the bony margin of the intervertebral foramen or the transverse process of the C-6 vertebral body.

The passage of the $\mathrm{cC}-7$ behind the esophagus was prepared earlier during exploration of the injured side. The development of this plane is completed from the normal contralateral side at this stage, and the $\mathrm{cC}-7$ is passed across the midline under direct inspection from both sides. Although Wang et al. recommend passage deep to the vertebral vessels, ${ }^{12}$ we opted to forego this challenging step to avoid injury to these vessels. The ultimate reach of the cC-7 across the midline could only be determined at this stage. Thus, the repair site of the lower trunk to the $\mathrm{cC}-7$ could be at the medial border of the ipsilateral scalenus anterior muscle or further medially in the retropharyngeal space.

Proximal mobilization of the lower trunk is completed at this stage. If shortening of the humerus is deemed necessary, the dissection is carried lateral to the biceps muscle, and the shaft is exposed. The humerus is osteomized transversely between the insertions of the deltoid and pectoralis major muscles. A segment of the shaft is excised from the distal side, and the bone ends are approximated. The length of the segment excised is determined by the difficulty experienced in attempting to approximate the 2 nerve ends (the transferred cC-7 and the ipsilateral lower trunk), varying from 2.5 to $3.5 \mathrm{~cm}$. The bone ends are held together, and fixation is performed using a 7- or 8-holed dynamic compression plate (Fig. 2). The resulting laxity of the biceps muscle is addressed by attempting to tighten the tendons of the 2 heads of the biceps with purse-string nonabsorbable sutures. The ulnar nerve is freed farther up to the level of the osteotomy. The site of entry of the superior ulnar collateral vessels limits the proximal migration of the lower trunk. These vessels must be preserved to retain the perfusion of the proximal ulnar nerve.

As this is not the only procedure being undertaken in such an injury, other nerve transfers are all completed prior to the final coaptation of $\mathrm{cC}-7$ to the lower trunk. In these cases, our typical reconstruction included nerve transfers of the medial cutaneous nerve of the forearm to the musculocutaneous nerve, the spinal accessory nerve 


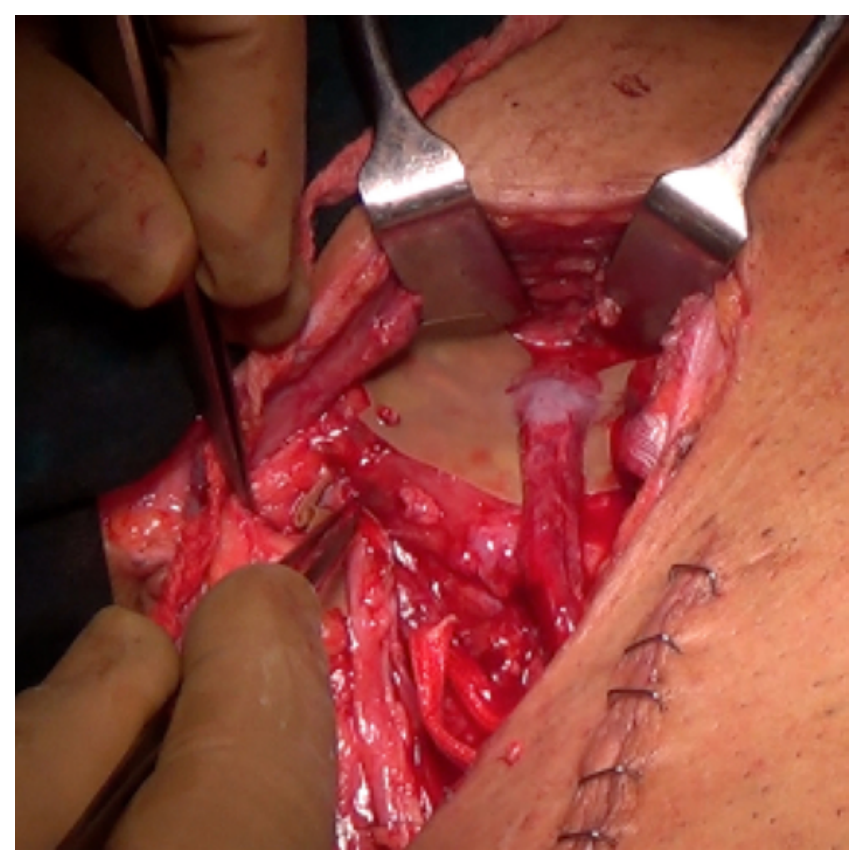

FIG. 3. Intraoperative photograph. Contrary to traditional teaching, a relaxed repair is not undertaken. Instead, this repair is performed under tension to avoid interposing grafts, thereby decreasing the loss of axons, as can occur at the sites of graft interposition.

to the suprascapular nerve, and the phrenic nerve directly to the posterior division of the lower trunk, for triceps muscle and finger extension functions. Once each of these transfers is successfully completed, the final maneuver to facilitate bringing the lower trunk directly to the $\mathrm{cC}-7$ is to fully adduct the arm across the chest, essentially bringing the hand to the contralateral shoulder. When the nerve ends are in a position for repair, the sternocleidomastoid muscle and the carotid sheath are retracted to facilitate visualization. The repair is performed using $6-0$ or 7-0 nylon to approximate these structures and relieve the tension at the repair site. The repair is completed with epineural sutures using 8-0 nylon (Fig. 3).

Obviously, such an extreme position in which nerves are under some tension requires immobilization to avoid rupturing the repairs in the early postoperative period. The first 2 patients received a brace holding the arm and forearm in front of the chest and a ring at the level of the forehead, preventing movements of the head, as proposed by Wang et al. ${ }^{24}$ This was maintained for 6 weeks followed by support in a sling for 2 more weeks. During this period, passive range of motion was maintained with intermittent flexion of the elbow and mobilization of the forearm, wrist, and hand. We later determined that movement of the head did not affect the repair and were able to simplify the immobilization. We therefore moved to a stockinette sling holding the affected arm adducted across the chest with the elbow in $120^{\circ}$ of flexion with the hand on the opposite shoulder, which was much simpler and sufficient to maintain our repair.

As discussed above, in cases for which we determined that we would not be successful in achieving the direct repair, we resorted to grafting. To accomplish this, the $\mathrm{cC}-7$ was delivered back to the normal side where suturing the nerve graft cables was technically less challenging. The cC-7 with its associated cable grafts was then carefully delivered back across the neck. We therefore eliminated the dramatic positioning that was previously used to ease tension on the repairs. The cables were typically $6-10 \mathrm{~cm}$ long, and the distal repair site was immediately proximal or deep to the clavicle. Obviously, the stump of the lower trunk could be trimmed with greater impunity in these cases. The repairs did not require tensioning sutures and were performed with 8-0 nylon alone. In such cases, the affected arm was supported in an elbow pouch sling for a month.

\section{Evaluation}

The patients were examined at 3-month intervals. Retraining exercises were instituted to strengthen the supraspinatus and biceps muscles as these functions emerged. The progression of the Tinel sign (in the distribution of the cC-7 on the donor limb) was followed along the deltopectoral region and into the affected arm. The patients were instructed to practice adduction of the opposite arm by pressing against the thigh while sitting.

The patients were instructed to report once wrist flexion was first noted. Instructions for strengthening were determined based on the activity that produced maximal flexion of the wrist and fingers.

At the latest follow-up, the strength of the flexor carpi ulnaris muscle and of the finger flexors was documented using the modified British Medical Research Council (MRC) muscle grading system. While we would have preferred objective force measurements, this was not feasible in our clinic setting. In addition, associated maneuvers required to accomplish the wrist and hand movement were noted. For example, some patients required forced synchronous activation of the opposite pectoralis major, latissimus dorsi, and triceps muscles to produce movement of the paralyzed hand. The final movements achieved were synkinetic, and wrist and finger flexion could not be activated separately. As a result, thumb and finger flexion could only be visualized by physically restraining the wrist. Again, electrodiagnostic tests were not considered helpful in the outcomes assessments because, regardless of the size or number of motor units achieved, ultimate strength and ease of activation provided more useful information.

\section{Results}

The median follow-up period was 26 months (range 23-39 months) for the direct coaptation group and 28.5 months (range 23-36 months) for the nerve graft group. Recovered function followed the trend described by Wang et al. ${ }^{24}$ with wrist flexion being strongest, followed by finger flexion and lastly by thumb flexion. At the final assessment, wrist and finger flexion were typically similar in strength, and thus our MRC scoring relates to these together or the score for the weaker of the muscles if there was disparity. Thus, 10 of the 12 patients the direct coaptation group demonstrated Grade 3 finger and wrist flexion and 2 patients had Grade 2 flexion. In the nerve graft 
TABLE 3. Comparison of finger and wrist flexion outcomes between the 2 groups

\begin{tabular}{ccc}
\hline $\begin{array}{c}\text { Finger \& Wrist } \\
\text { Flexion MRC Grade }\end{array}$ & $\begin{array}{c}\text { cC-7 Direct } \\
\text { Coaptation Group }\end{array}$ & $\begin{array}{c}\text { cC-7 Nerve } \\
\text { Graft Group }\end{array}$ \\
\hline 3 & $10(83.3 \%)$ & $2(20 \%)$ \\
\hline 2 & $2(16.6 \%)$ & $7(70 \%)$ \\
\hline 0 & - & $1(10 \%)$ \\
\hline
\end{tabular}

group, only 2 of 10 patients achieved Grade 3, whereas 7 had Grade 2, and 1 had Grade 0 flexion (Table 3).

In the direct repair group, all patients achieved some flexion of the wrist and fingers. The function typically appeared by 12-14 months after the operation and improved rapidly. One patient showed this function for the first time at 24 months. The patients typically activated this function with mild adduction of the donor arm. Function of the flexor carpi ulnaris muscle and flexor digitorum profundus muscles of the middle, ring, and little fingers was consistently documented, and these tended to be the strongest muscles restored. Additionally, many achieved function of the index flexor digitorum profundus and flexor pollicis longus muscles as well (Figs. 4 and 5, and Videos 1 and 2).

VIDEO 1. Video clip demonstrating a typical case after the use of grafts to connect the $\mathrm{cC}-7$ with the ipsilateral lower trunk. This illustrates the required forced adduction of the contralateral arm to produce the relatively weak flexion of the reconstructed hand. Compare this with the function achieved and the lack of forced contralateral activity in the no graft reconstruction. Copyright Deenanath Mangeshkar Hospital. Published with permission. Click here to view.

VIDEO 2. Video showing direct repair at 38 months following wrist fusion. With immobilization of the wrist, the action of the fingers becomes more obvious. Both active finger flexion and extension are achieved by the combination of $\mathrm{cC}-7$ and ipsilateral phrenic transfers. Copyright Deenanath Mangeshkar Hospital. Published with permission. Click here to view.

In the nerve grafting group, some flexion of the wrist and fingers was noted in 9 of the 10 patients. However, these functions appeared at 16-18 months, a 3- to 6-month delay when compared with the direct repair group. In most of these patients, the strength did not progress, and considerable effort was required on the opposite side to achieve the movement (Fig. 6 and Video 3).

VIDEO 3. Video showing direct repair of $\mathrm{cC}-7$ at 36 months. It is apparent that this patient can activate relatively robust wrist flexion. Finger flexion is also present but less obvious due to the action of the wrist. Copyright Deenanath Mangeshkar Hospital. Published with permission. Click here to view.

In 1 patient, no function could be elicited in the forearm flexors even at 2 years after the operation.

Sensation over the ulnar half of the hand was not evaluated in detail. Of note, the lateral cord contribution of the median nerve is not reinnervated in this technique, so sensation over the thumb and index finger is not expected. The patients, though, did report an improvement in sensation with the passage of time (starting at 16-18 months from the operation).

\section{Complications}

Each patient described paresthesias in the distribution

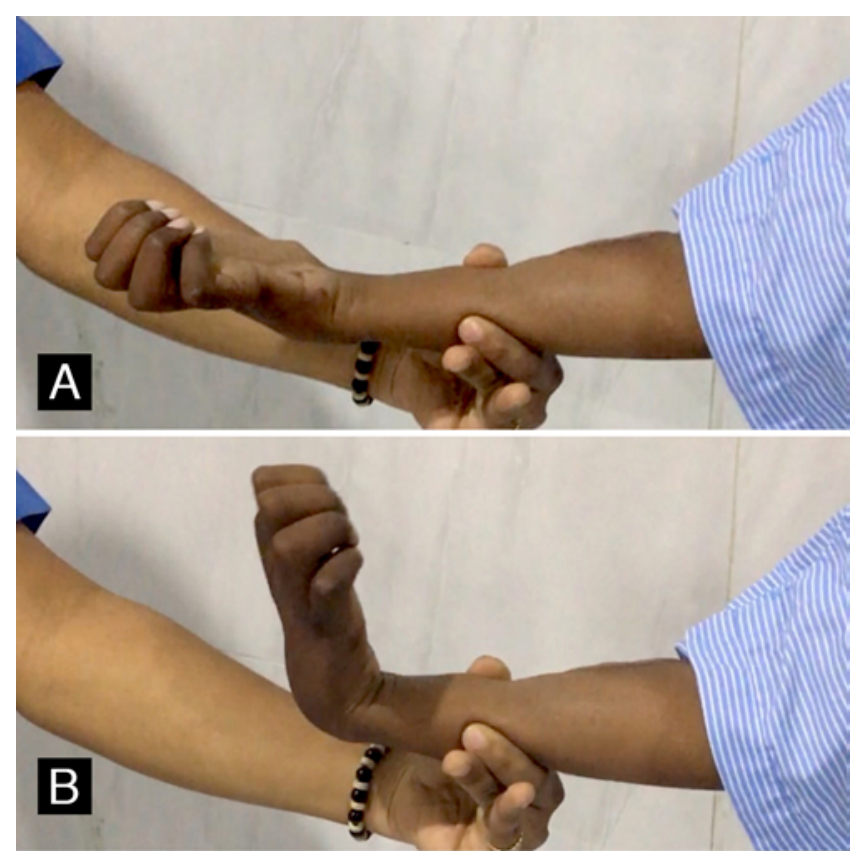

FIG. 4. At 36 months after direct CC-7 repair from the $\mathrm{CC}-7$ to the lower trunk, from a neutral position (A), this patient is able to flex her wrist well, easily overcoming gravity (B). This is done without effort from the contralateral limb.

of the $\mathrm{cC}-7$ root (pulps of the thumb and index finger) immediately after surgery. This disappeared over the course of 1-6 months after the operation. In addition, relative weakness of the triceps muscle could be documented in some patients. However, no complete deficits were encountered. No patient in either group complained of any enduring deficit in the donor limb at long-term follow-up. This was also not objectively recorded. Only the patient's subjective experience of deficit was assessed.

One patient in the nerve graft group had a transient bilateral phrenic nerve deficit. The patient had sustained a phrenic nerve injury at the time of the brachial plexus injury and had unilateral diaphragmatic paralysis on the side of his brachial plexus injury prior to surgery. Unfortunately, when the $\mathrm{cC}-7$ was delivered from the healthy side to the injured side, there was unintentional traction on the healthy phrenic nerve. This resulted in a neurapraxic injury, and the patient required a tracheostomy and assisted ventilation for 10 days. The diaphragm demonstrated return of adequate excursion at this time. The patient was thus weaned from the ventilator and discharged 2 weeks after the operation.

One patient in the direct repair group experienced persistent lymphatic drainage from the wound over the healthy side of the neck (strangely, it was the right side). The wound did not fully close for 2 months. No patient in either group experienced any obvious weakness or deficit in the donor upper limb following the operation. Six patients described paresthesias in the thumb and index finger that disappeared over 1-6 months.

One patient in the direct repair group experienced anteroinferior dislocation of the humerus from the glenohumeral joint after the stockinette sling was removed. There 


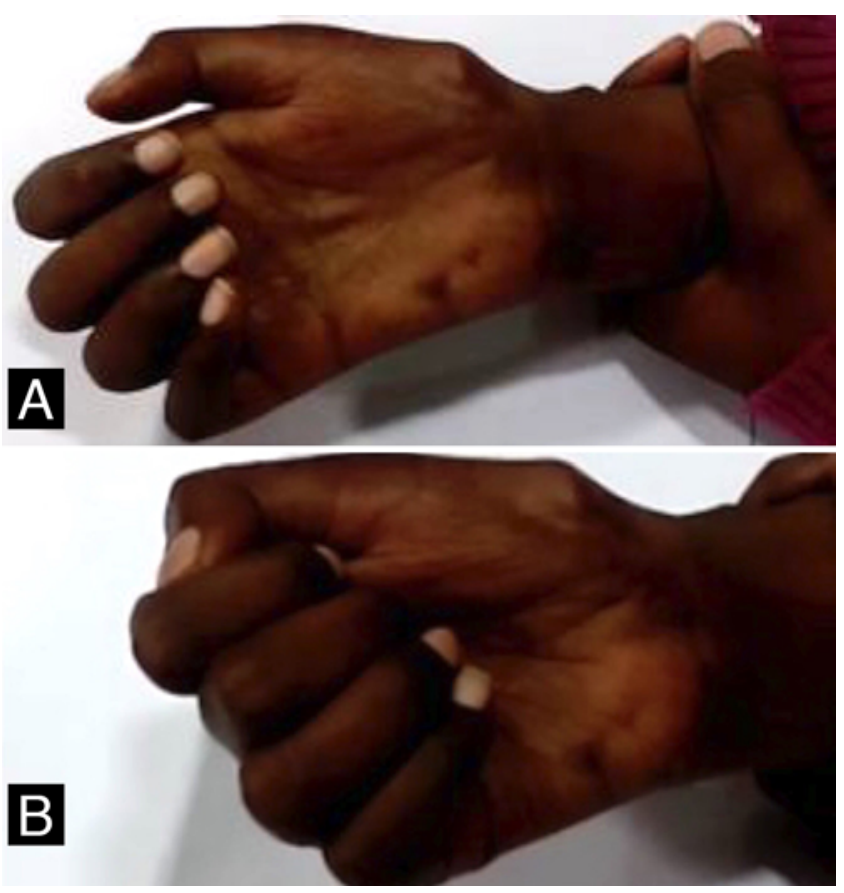

FIG. 5. After immobilization of the wrist, action of the fingers becomes more apparent. A: The active extension of the fingers is driven by the phrenic to the posterior division of the upper trunk. B: The active finger flexion is driven by the direct repair from the $\mathrm{CC}-7$ to the anterior division of the lower trunk. Again, minimal action of the contralateral limb is required for this activation.

was no past history of injury to the affected shoulder. The dislocation interfered with activation of the recovering biceps and triceps muscles. Contractions of these muscles were evident only after physical reduction of the head of the humerus. At 2 years after surgery, the shoulder remained stable in the reduced position, and the patient recovered function in the supraspinatus muscle (from the spinal accessory nerve-to-suprascapular nerve transfer).

\section{Discussion}

Treatment of complete brachial plexus injuries remains a challenge to all peripheral nerve specialists. The effectiveness of nerve transfer procedures for consistent restoration of basic proximal functions has been shown. ${ }^{15,21}$ However, effective restoration of prehension has, so far, eluded us. The cC-7 transfer offers the most promising option for restoring finger flexion in a consistent manner. The cC-7 transfer provides the greatest quantity of myelinated nerve fibers of any potential nerve transfer, ${ }^{2,7}$ and it is logical to expect better distal function when a larger number of axons are directed toward the target muscles. This is clearly evident from the difference in the rates of success between reports of hemi-cC7 and full cC-7 transfers., $3,8,16,20$

The prespinal route serves to shorten the gap between the donor and recipient nerves..$^{2}$ Prespinal passage must be performed under direct visualization, which requires a trained surgeon working from either side of the neck. As noted above, 1 patient in this series suffered incidental traction on the phrenic nerve of the uninjured side. In our series, the repair site was often closer to the midline than the medial margin of the ipsilateral anterior scalene muscle. In addition, the zeal for a direct repair had to be tempered by the need to trim the recipient $\mathrm{C}-8$ and $\mathrm{T}-1$ roots until the ends appeared healthy under the microscope. Nerve grafting is technically easier and avoids shortening of the humerus. However, after observing the outcomes,
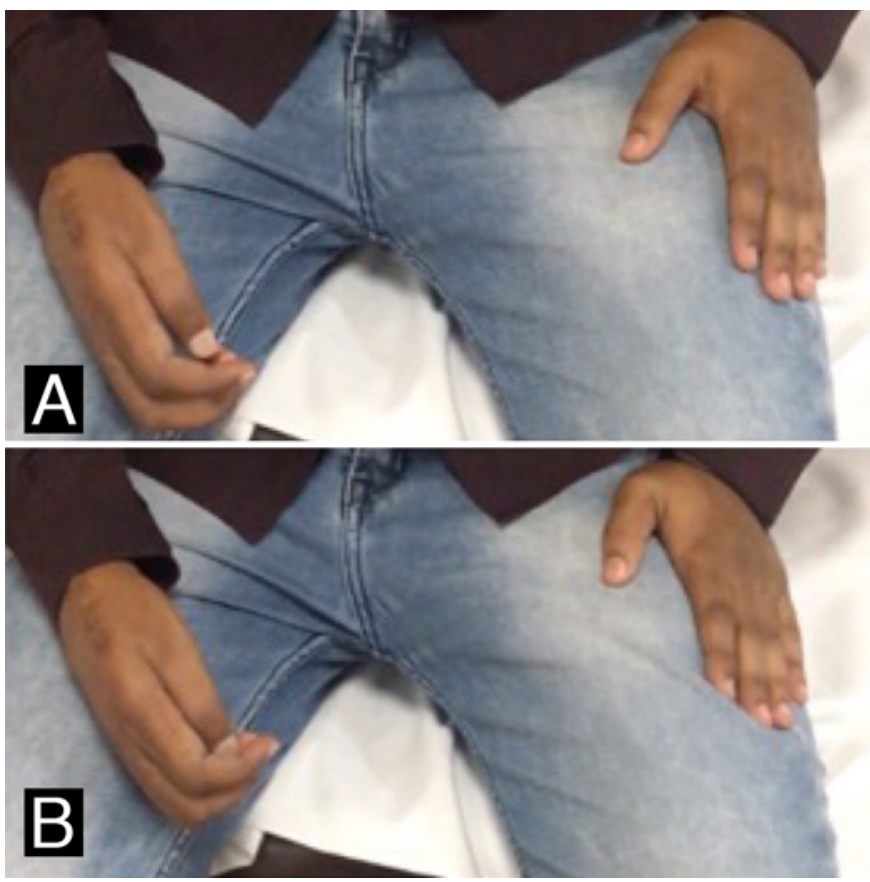
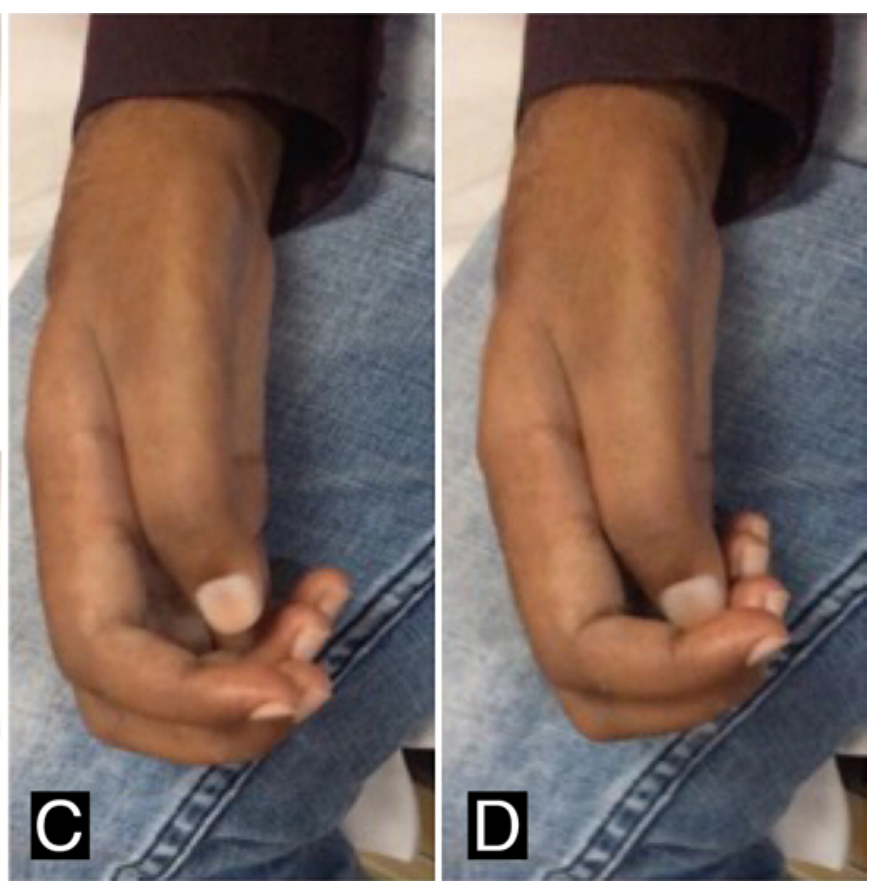

FIG. 6. A and B: With interposed grafts, the flexion of the fingers is driven by pressing the contralateral arm into the thigh as a method of driving strong adduction (B). C and D: The finger flexion achieved is much less robust than that afforded by direct repair. 
we shifted our policy to performing direct coaptation of the $\mathrm{cC}-7$, if possible.

The conclusion reached in this small study is contradictory to commonly accepted dogma in peripheral nerve surgery, which dictates that tension at a repair site should be avoided at all costs and accounts for poor outcomes. ${ }^{13,18}$ This study can be added to mounting recent clinical and basic science findings that contradict this assertion., ${ }^{9,22}$ While severe traction is clearly injurious to a peripheral nerve, it is becoming increasingly clear that moderate traction will, in fact, promote neuronal growth. Alternatively, in the presence of nerve grafts, fewer axons would be conveyed to the target and in a delayed fashion. This is the consequence of axons navigating 2 repair sites and the staggered regeneration that occurs at each, with a significant subset of axons failing to enter an endoneurial tube and being conveyed either through the graft or from the graft into the distal nerve. ${ }^{10}$

To date, the effect of using nerve grafts instead of direct coaptation of $\mathrm{cC}-7$ had not been evaluated. In fact, the report by Feng et al. in which a different subcutaneous repair (passage around the sternocleidomastoid muscle) was performed appears to be the only comparable study in which the effect of nerve grafting in such a situation was described. ${ }^{6}$

Our current study provided us with a unique opportunity to see the quality of reinnervation of the forearm flexors following direct repair of the $\mathrm{cC}-7$ to the lower trunk and following interposition of nerve grafts between the same donor and recipient nerves. Patient characteristics, including the age and preoperative delay, in the 2 groups were similar. The patients exhibited flexion of the wrist and fingers at 12-14 months after the operation for the direct repairs and at 16-18 months in the nerve graft group. The recipient function could be elicited with less effort of the donor limb, strengthened more rapidly, and the effort required was reduced over time. This applied uniformly to all patients in whom a direct repair was performed. However, nerve grafting resulted in weaker flexion of the target wrist and fingers. The strength reached a plateau at an early stage (within 6 months after appearance) and ultimately necessitated exertion of considerable effort in the donor upper limb.

The recipient functions are initially demonstrated by exerting the original function served by the donor nerve, as with every nerve transfer. ${ }^{11}$ Thus, the patient is instructed to adduct the arm to activate the pectoralis major and latissimus dorsi muscles and to extend the elbow against resistance for the triceps muscle. However, the patients did not achieve any independence of the different forearm functions in the reconstructed limb. Flexion of the fingers and of the wrist happened simultaneously in each case. The branches of the ulnar nerve to the flexor carpi ulnaris muscle and those of the median and ulnar nerves to the flexor digitorum superficialis/flexor digitorum profundus muscles are just 1-2 inches apart. ${ }^{23}$ The appearance of these functions is proof of the arrival of growing axons in sufficient numbers and confirms the integrity of the repair done by approximation of the nerve ends. It is not practical to express the result separately in terms of strength in the flexors of the wrist and of the fingers.
We had concerns about the effect of shortening the humerus on the ultimate biceps muscle strength. However, we have not observed a significant reduction in the success rate for restoration of elbow flexion.

It appears that a reliable intervention has been devised to restore function in the wrist and fingers. How this translates into active function and its impact on activities of daily living are the next steps in this endeavor. Once we establish that we can reliably reanimate muscles, we can then move on to optimize the function achieved. Possible next steps would include transfer of the flexor carpi ulnaris to provide wrist extension so that the synergy of wrist extension and finger flexion is capitalized upon. Additionally, a static intrinsic reconstruction would position the fingers more optimally to allow for key pinch and grasp.

\section{Conclusions}

We report this study to validate this challenging technique that appears to be reliable in restoration of extrinsic flexion of the fingers. At this point, we reserve this procedure for highly motivated patients who have limited comorbidities and (given our complication) documented intact bilateral phrenic nerve function. This procedure should be undertaken by a surgeon who performs brachial plexus reconstruction as a major part of his/her practice. For us, our results have served as sufficient evidence to make every effort to achieve a direct approximation of the opposite C-7 with the ipsilateral lower trunk. In fact, if such a coaptation is not possible, we prefer to avoid use of the $\mathrm{cC}-7$ altogether.

\section{References}

1. Bahm J, Ocampo-Pavez C: Free functional gracilis muscle transfer in children with severe sequelae from obstetric brachial plexus palsy. J Brachial Plex Peripher Nerve Inj 3:23, 2008

2. Chuang DC: Neurotization procedures for brachial plexus injuries. Hand Clin 11:633-645, 1995

3. Chuang DCC, Hernon C: Minimum 4-year follow-up on contralateral C7 nerve transfers for brachial plexus injuries. J Hand Surg Am 37:270-276, 2012

4. Doi K, Kuwata N, Muramatsu K, Hottori Y, Kawai S: Double muscle transfer for upper extremity reconstruction following complete avulsion of the brachial plexus. Hand Clin 15:757767, 1999

5. Doi K, Muramatsu K, Hattori Y, Otsuka K, Tan SH, Nanda V, et al: Restoration of prehension with the double free muscle technique following complete avulsion of the brachial plexus. Indications and long-term results. J Bone Joint Surg Am 82:652-666, 2000

6. Feng J, Wang T, Gu Y, Chen L, Zhang G, Zhu Y: Contralateral C7 transfer to lower trunk via a subcutaneous tunnel across the anterior surface of chest and neck for total root avulsion of the brachial plexus: a preliminary report. Neurosurgery 66 (6 Suppl Operative):252-263, 2010

7. Gu Y, Xu J, Chen L, Wang H, Hu S: Long term outcome of contralateral C7 transfer: a report of 32 cases. Chin Med J (Engl) 115:866-868, 2002

8. Gu YD, Chen DS, Zhang GM, Cheng XM, Xu JG, Zhang LY, et al: Long-term functional results of contralateral C7 transfer. J Reconstr Microsurg 14:57-59, 1998

9. Hara Y, Nishiura Y, Ochiai N, Sharula, Nakajima Y, Kubota $\mathrm{S}$, et al: New treatment for peripheral nerve defects: recon- 
struction of a $2 \mathrm{~cm}$, monkey median nerve gap by direct lengthening of both nerve stumps. J Orthop Res 30:153-161, 2012

10. Hentz VR, Rosen JM, Xiao SJ, McGill KC, Abraham G: The nerve gap dilemma: a comparison of nerves repaired end to end under tension with nerve grafts in a primate model. J Hand Surg Am 18:417-425, 1993

11. Kahn LC, Moore AM: Donor activation focused rehabilitation approach: maximizing outcomes after nerve transfers. Hand Clin 32:263-277, 2016

12. Mcguiness CN, Kay SP: The prespinal route in contralateral C7 nerve root transfer for brachial plexus avulsion injuries. J Hand Surg Br 27:159-160, 2002

13. Millesi H: The nerve gap. Theory and clinical practice. Hand Clin 2:651-663, 1986

14. Narakas AO, Hentz VR: Neurotization in brachial plexus injuries. Indication and results. Clin Orthop Relat Res (237):43-56, 1988

15. Rinker B: Nerve transfers in the upper extremity: a practical user's guide. Ann Plast Surg 74 (Suppl 4):S222-S228, 2015

16. Sammer DM, Kircher MF, Bishop AT, Spinner RJ, Shin AY: Hemi-contralateral C7 transfer in traumatic brachial plexus injuries: outcomes and complications. J Bone Joint Surg Am 94:131-137, 2012

17. Songcharoen P, Wongtrakul S, Mahaisavariya B, Spinner RJ: Hemi-contralateral C7 transfer to median nerve in the treatment of root avulsion brachial plexus injury. J Hand Surg Am 26:1058-1064, 2001

18. Terzis J, Faibisoff B, Williams B: The nerve gap: suture under tension vs. graft. Plast Reconstr Surg 56:166-170, 1975

19. Terzis JK, Kostopoulos VK: The surgical treatment of brachial plexus injuries in adults. Plast Reconstr Surg 119:73e92e, 2007

20. Tu YK, Tsai YJ, Chang CH, Su FC, Hsiao CK, Tan JSW: Surgical treatment for total root avulsion type brachial plexus injuries by neurotization: a prospective comparison study between total and hemicontralateral C7 nerve root transfer. Microsurgery 34:91-101, 2014

21. Tung TH: Nerve transfers. Clin Plast Surg 41:551-559, 2014

22. Vaz KM, Brown JM, Shah SB: Peripheral nerve lengthening as a regenerative strategy. Neural Regen Res 9:1498-1501, 2014

23. Wang B, Li H, Zhang J, Yang H, Liu W, Jiang W, et al: [Anatomical study on contralateral C7 root transfer for recovery of forearm flexor function in repairing of brachial plexus avulsion.] Zhongguo Xiu Fu Chong Jian Wai Ke Za Zhi 26:1223-1226, 2012 (Chinese)

24. Wang SF, Li PC, Xue YH, Yiu HW, Li YC, Wang HH: Contralateral $\mathrm{C} 7$ nerve transfer with direct coaptation to restore lower trunk function after traumatic brachial plexus avulsion. J Bone Joint Surg Am 95:821-827, S1-S2, 2013

25. Xu WD, Lu JZ, Qiu YQ, Jiang S, Xu L, Xu JG, et al: Hand prehension recovery after brachial plexus avulsion injury by performing a full-length phrenic nerve transfer via endoscopic thoracic surgery. J Neurosurg 108:1215-1219, 2008

26. Yang G, Chang KW, Chung KC: A systematic review of contralateral C7 transfer for the treatment of traumatic brachial plexus injury: part 1. Overall outcomes. Plast Reconstr Surg 136:794-809, 2015

\section{Disclosures}

The authors report no conflict of interest concerning the materials or methods used in this study or the findings specified in this paper.

\section{Author Contributions}

Conception and design: Bhatia. Acquisition of data: Bhatia, Doshi, Koul, Shah. Analysis and interpretation of data: Brown, Salama. Drafting the article: Bhatia, Doshi, Koul. Critically revising the article: Koul, Brown, Salama. Reviewed submitted version of manuscript: Shah. Statistical analysis: Salama. Administrative/ technical/material support: Doshi, Koul, Shah, Brown, Salama. Study supervision: Bhatia, Shah.

\section{Supplemental Information \\ Videos}

Video 1. https://vimeo.com/216829388.

Video 2. https://vimeo.com/216829502.

Video 3. https://vimeo.com/216829620.

\section{Correspondence}

Anil Bhatia, Department of Brachial Plexus Surgery, Deenanath Mangeshkar Hospital, Nal Stop, Kothrud, Pune 411004, India. email: agbhatia63@gmail.com. 